\title{
Riqueza de Agaricomycetes poroides da Serra do Navio, Amazônia oriental, com novo registro de Oxyporus lacera para o Brasil \\ Richness of poroid Agaricomycetes from Serra do Navio, eastern Amazonia, Brazil, with a new record of Oxyporus lacera for Brazil
}

\author{
William Kalhy Silva Xavier', Helen Maria Pontes Sotão", Adriene Mayra da Silva Soares", \\ Tatiana Baptista Gibertonill', Felipe de Jesus Rodrigues', Leif Ryvarden ${ }^{\text {IV }}$ \\ 'Universidade do Estado do Amapá. Macapá, Amapá, Brasil \\ IIMuseu Paraense Emílio Goeldi/MCTIC. Belém, Pará, Brasil \\ '"'Universidade Federal de Pernambuco. Recife, Pernambuco, Brasil \\ IV University of Oslo. Oslo, Noruega
}

\begin{abstract}
Resumo: Os Agaricomycetes poroides são macrofungos caracterizados pela presença do himenóforo tubular, estrutura que se apresenta em forma de poros quando vista frontalmente. Este trabalho apresenta as espécies de Agaricomycetes poroides coletadas entre outubro de 2014 e junho de 2017 em áreas florestais da Serra do Navio, no estado do Amapá, Amazônia brasileira. A partir de análises morfológicas dos espécimes coletados, 100 espécies de Agaricomycetes foram identificadas, distribuídas em 54 gêneros, dez famílias e três ordens (Hymenochaetales, Polyporales e Russulales). Todas as espécies são primeiros registros para a área de estudo, sendo 18 novos registros para o Amapá e quatro para a Amazônia brasileira, além de Oxyporus lacera Ryvarden, que representa o primeiro registro para o Brasil. São apresentados comentários taxonômicos, distribuição e ilustração para esta espécie. Considerando-se a frequência relativa, 79\% das espécies foram analisadas como raras, $20 \%$ como ocasionais e apenas $1 \%$ foi considerada como frequente. Este estudo expande o conhecimento sobre a distribuição geográfica dessas espécies para a Amazônia brasileira.
\end{abstract}

Palavras-chave: Fungos. Basidiomycota. Hymenochaetales. Polyporales. Russulales. Amapá.

\begin{abstract}
This work presents the poroid Agaricomycetes found in forest areas of Serra do Navio, state of Amapá, in the Brazilian Amazon. Taxonomic study and morphological analyses of collected specimens resulted in the identification of 100 species of Agaricomycetes, distributed in 54 genera, ten families, and three orders (Hymenochaetales, Polyporales, and Russulales). All species represent the first records for the study area, and 18 are new records for the state of Amapá, four are new for the Brazilian Amazon and Oxyporus lacera Ryvarden is reported for the first time for Brazil. Taxonomic comments, geographic distributions, and illustrations of this species are provided herein. As for the relative frequency, about $79 \%$ of the species were considered rare, $20 \%$ were occasional, and only $1 \%$ were considered frequent. This study increases our knowledge of the geographic distribution of these species in the Brazilian Amazon.
\end{abstract}

Keywords: Fungi. Basidiomycota. Hymenochaetales. Polyporales. Russulales. Amapá.

XAVIER, W. K. S., H. M. P. SOTÃO, A. M. S. SOARES, T. B. GIBERTONI, F. J. RODRIGUES \& L. RYVARDEN, 2018. Riqueza de Agaricomycetes poroides da Serra do Navio, Amazônia oriental, com novo registro de Oxyporus lacera para o Brasil. Boletim do Museu Paraense Emílio Goeldi. Ciências Naturais 13(3): 303-315.

Autora para correspondência: Helen Maria Pontes Sotão. Museu Paraense Emílio Goeldi/MCTIC. Coordenação de Botânica. Av. Perimetral, 1901 - Terra Firme. Belém, PA, Brasil. CEP 66077-530 (helen@museu-goeldi.br).

Recebido em 16/05/2018

Aprovado em 28/09/2018

Responsabilidade editorial: Fernando da Silva Carvalho Filho

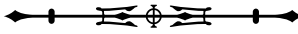




\section{INTRODUÇÃO}

Agaricomycetes são fungos pertencentes ao filo Basidiomycota e caracterizam-se pela produção exógena de esporos sexuais (basidiósporos) em basídios, geralmente originados em estruturas macroscópicas denominadas de basidiomas, podendo apresentar doliporos perfurados ou não perfurados (Hibbett et al., 2014). A presença de himenóforo tubular configura-se como característica peculiar de parte dos indivíduos desta classe, estrutura que se parece com poros quando vista frontalmente, por essa razão, os indivíduos são também chamados de poliporos ou de fungos poroides (Ryvarden, 1991; Robledo \& Rajchenberg, 2007).

No Brasil, são catalogadas 5.719 espécies de fungos, das quais 301 são de Agaricomycetes poroides listadas para o Norte do Brasil (Gomes-Silva \& Gibertoni, 2009; Soares et al., 2014; JBRJ, s. d.). Trabalhos publicados na última década contendo inventários taxonômico e ecológico de áreas pouco exploradas da Amazônia brasileira - como os realizados na Floresta Nacional (FLONA) de Caxiuanã, no estado do Pará, por Sotão et al. (2009), Gibertoni et al. (2013) e Medeiros et al. (2013), que registraram 87, 96 e 76 espécies respectivamente - contribuíram de maneira significativa para o atual número de registros conhecidos para esta região.

Para o estado do Amapá, apenas 103 espécies de fungos poroides são conhecidas. Sotão et al. (1991, 2003) listam 33 espécies de macrofungos em ecossistemas de manguezais do litoral, todas representando o primeiro registro para o estado, entre as quais 22 são poroides. Soares et al. (2014) listam 97 espécies de Agaricomycetes poroides em área de floresta ombrófila densa da FLONA do Amapá, entre as quais 77 são novos registros para este estado; mais recentemente, quatro novas espécies desta área foram descritas por Hyde et al. (2017) e Tibpromma et al. (2017). Esses dados representam um número baixo, considerando-se o potencial da biodiversidade da Amazônia.

O presente estudo teve como objetivo ampliar o conhecimento sobre Agaricomycetes poroides para a
Amazônia brasileira, a partir de um inventário realizado na Serra do Navio.

\section{MATERIAL E MÉTODOS}

A vegetação predominante no município de Serra do Navio, no estado do Amapá (00 53' 44” N e 5200” 08” W), é formada por floresta ombrófila densa, com clima tropical e com alta taxa anual de precipitação $(2.000 \mathrm{~mm})$. Possui relevo montanhoso, com elevação de 63 a 303 m. Esta região sofreu intenso processo de degradação devido à extração mineral de manganês, resultando em um mosaico de vegetação nativa e recuperada. $\mathrm{Na}$ área da sede do município, na cidade de Serra do Navio, está instalado o Parque do Cancão, uma unidade de conservação municipal localizada na cidade, com vegetação de floresta nativa (Drummond \& Pereira, 2007).

Entre outubro de 2014 e junho de 2017, foi realizada uma viagem a campo a cada quatro meses, totalizando oito coletas. Os basidiomas foram coletados em 18 parcelas de 250 × 10 m, com uma distância mínima de 500 m entre elas. Três dessas parcelas estavam no Parque do Cancão e as demais, em diferentes pontos selecionados no município de Serra do Navio (Figura 1).

As técnicas determinadas por Fidalgo \& Bononi (1989) foram utilizadas para a coleta e a preservação dos basidiomas poroides. A análise das microestruturas foi realizada a partir de cortes feitos à mão livre com lâminas de aço, posicionados entre lâminas e lamínulas, com solução de $\mathrm{KOH} 3 \%$ (hidróxido de potássio) e floxina 1\%, ou por meio de reagente de Melzer, para observar reação dextrinoide ou amiloide (Teixeira, 1995).

Para a identificação e/ou confirmação das espécies, foi utilizada literatura especializada, como as obras de Ryvarden \& Johansen (1980), Ryvarden \& Gilbertson (1993), Ryvarden \& Melo (2014) e Ryvarden (1991, 2004, 2007, 2014, 2016). O material coletado foi depositado no Herbário MG, do Museu Paraense Emílio Goeldi (MPEG), e no Herbário Amapaense (HAMAB), do Instituto de Pesquisas Científicas e Tecnológicas do Estado do Amapá (IEPA). 


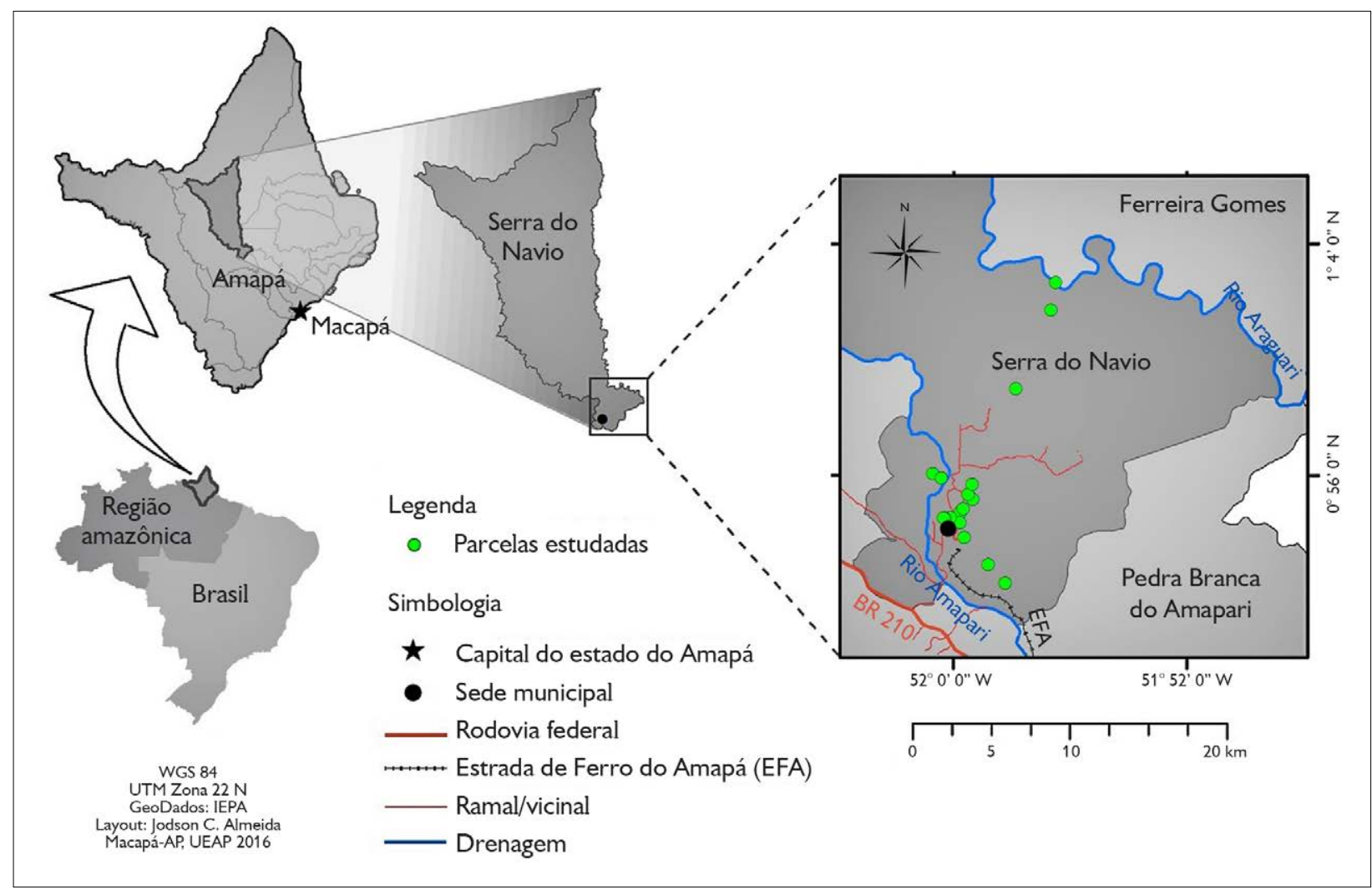

Figura 1. Locais de coletas de fungos Agaricomycetes poroides no município de Serra do Navio, estado do Amapá, Brasil. Mapa: Jodson C. Almeida (2018).

A frequência relativa dos fungos poroides $(F)$ foi determinada pela fórmula $F=(n \times 100) / N$, em que $n$ é o número de espécimes de uma determinada espécie de fungo e $N$ é o número total de espécimes encontrados. Para isso, foram adotadas as classes de frequência indicadas nos estudos de Lindblad (2000) e Hattori (2005), sendo: $0,5<F \leq 1,5 \%=$ rara; $1,5<F \leq 5 \%=$ ocasional; $5<$ $F \leq 10 \%=$ frequente; $F>10 \%=$ abundante.

A fim de avaliar a suficiência amostral e de estimar a riqueza na área de estudo, foram aplicados os índices Chao 1 (primeira ordem), Chao 2 (segunda ordem) e Jackknife 1 e 2, por meio do programa EstimateS 8.0 (Colwell, 2009).

Com base nos estudos taxonômicos realizados, foi elaborada uma lista dos táxons identificados, organizada em ordem alfabética, apresentados no Apêndice, contendo a classe de frequência, o número de espécimes identificados, indicação do voucher depositado no herbário MG e distribuição da espécie no Brasil, seguindo JBRJ (s. d.), CRIA (s. d.) e outras literaturas indicadas. A classificação e a nomenclatura dos táxons citados estão de acordo com Justo et al. (2017) e/ou com Index Fungorum (2018).

\section{RESULTADOS E DISCUSSÃO}

Foi identificado total de 1.443 espécimes de Agaricomycetes poroides da Serra do Navio, representando 100 espécies, 54 gêneros e dez famílias de Hymenochaetales (Hymenochaetaceae, Schizoporaceae), Polyporales (Fomitopsidaceae, Ganodermataceae, Meripilaceae, Meruliaceae, Phanerochaetaceae, Polyporaceae, Steccherinaceae) e Russulales (Bondarzewiaceae) (Apêndice).

Polyporaceae Corda foi a família com maior número de representantes, com 26 gêneros e 46 espécies, 
seguida por Hymenochaetaceae (11 gêneros e 17 espécies) e Ganodermataceae (dois gêneros e sete espécies). Perenniporia Murrill foi o gênero com o maior número de espécies (nove), seguido por Trametes Fr. (seis) e Rigidoporus Murrill (cinco) (Apêndice).

As espécies com maior densidade foram Xylodon flaviporus (Berk. \& M.A. Curtis ex Cooke) Riebesehl \& Langer sensu lato (130) (Figura 2A), Trametes elegans (Spreng.) Fr. (80) (Figuras 2B e 2C), Polyporus leprieurii Mont. (73) (Figura 2D), Tinctoporellus epimiltinus (Berk. \& Broome) Ryvarden (72) (Figura 2E), Polyporus dictyopus Mont. sensu lato (64) (Figura 2F) e Ganoderma australe (Fr.) Pat. (63) (Figura 2G). Algumas dessas podem representar complexos de espécies (Paulus et al., 2000; Insumran et al., 2012; Ferreira-Lopes et al., 2016; Palacio et al., 2017), sendo necessárias ferramentas moleculares para sua melhor delimitação. Assim, tais espécies identificadas neste trabalho serão mantidas como sensu lato no Apêndice.

Considerando-se a análise da frequência relativa, 79\% das espécies (79) foram consideradas raras, 20\% (20) foram consideradas ocasionais e 1\% (X. flaviporus sensu lato) foi considerada frequente (Apêndice), resultado semelhante aos obtidos por Gibertoni et al. (2007), Gibertoni (2008), Medeiros et al. (2013) e Soares et al. (2014).

A estimativa de riqueza de espécies variou de 136 para Chao 1, e de 151 para Chao 2 e para Jacknife 2 (Figura 3). Os valores obtidos pelos estimadores de riqueza sugerem que $81 \%$ a $90 \%$ dos Agaricomycetes poroides foram coletados na área de estudo, mas também demonstram que, possivelmente, mais espécies serão encontradas, caso o período de amostragem seja estendido. Vale ressaltar que o estudo realizado por Soares et al. (2014) na FLONA do Amapá, região próxima à área do presente estudo, registrou 97 espécies de Agaricomycetes poroides, além de ter compilado valores de $85 \%$ para os mesmos estimadores de riqueza avaliados; enquanto Gibertoni et al. (2016) obtiveram $83,15 \%$ usando como estimador de riqueza Jacknife 1, em estudo realizado em três área de endemismo da Amazônia, incluindo o Amapá.

A riqueza encontrada nas áreas florestais do município de Serra do Navio aproxima-se àquelas observadas em outras áreas da Amazônia oriental, como evidenciaram os estudos de Soares et al. (2014), de Sotão et al. (2009), de Gibertoni et al. (2013) e de Medeiros et al. (2013), realizados na FLONA de Caxiuanã, que relataram 97, 87, 96 e 76 espécies, respectivamente. Esses trabalhos também citam a predominância de espécies da família Polyporaceae nos inventários amazônicos.

Todas as espécies identificadas representam o primeiro registro para a área de estudo. Dezoito espécies foram identificadas como novos registros para o estado do Amapá (Apêndice), quatro para a Amazônia [Ceriporia purpurea (Fr.) Donk (Figura 2H), Dichomitus campestris (Quél.) Domanski \& Orlicz, Perenniporia centrali-africana Decock \& Mosseb (Figura 2I) e Oxyporus latemarginatus (Durieu \& Mont.) Donk.] e uma para o Brasil (O. lacera Ryvarden).

Oxyporus lacera (Figura 4) é reconhecida por apresentar basidioma branco, ressupinado, com poros angulares a irregulares, lacerados e rasos (1-2 por $\mathrm{mm})$. Possui sistema hifálico monomítico, cistídios abundantes, basidiósporos elipsoides (3-4 × 2-2,5 $\mu \mathrm{m})$ (Ryvarden, 2007). No espécime analisado (MG226149), o número de poros variou entre 2-3 por $\mathrm{mm}$, e os basidiósporos mediram entre 3,5-4,5 × 2,0 $\mu \mathrm{m}$. Oxyporus pellicula (Jungh.) Ryvarden é uma espécie similar a 0 . lacera, entretanto apresenta basidiósporos maiores e amplamente elipsoides [4,5-6,5 (7) × 3-4,5 $\mu \mathrm{m})]$ (Ryvarden, 2007). Anteriormente, O. lacera era conhecida apenas para a localidade-tipo (Belize, Distrito de Cayo) (Ryvarden, 2007).

Considerando-se a escassez de informações sobre a ocorrência de macrofungos da micobiota amazônica, o presente trabalho contribui para o conhecimento da riqueza de fungos poroides procedentes de inventário em áreas florestais previamente não amostradas na Amazônia.

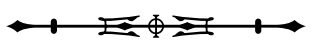



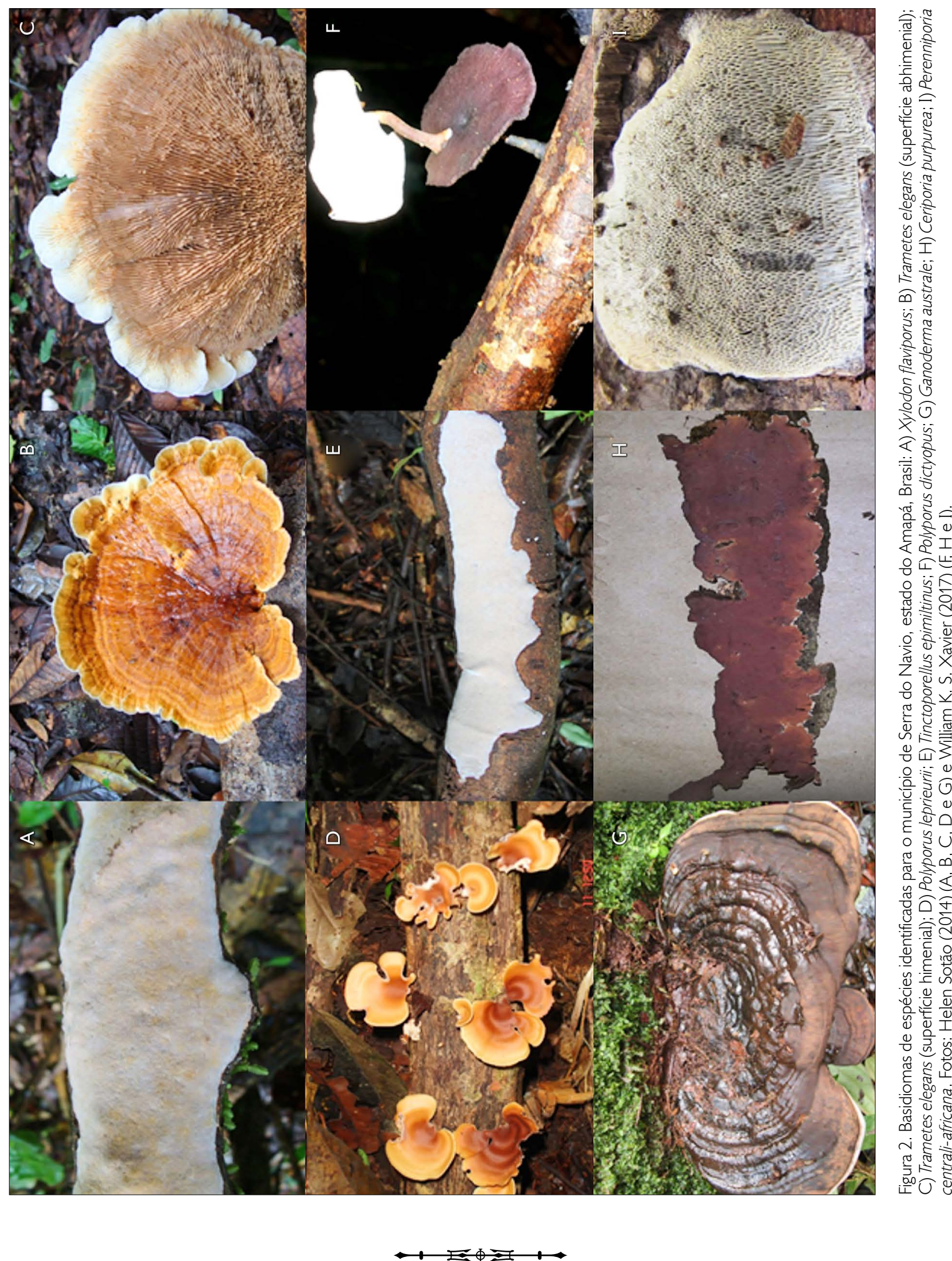


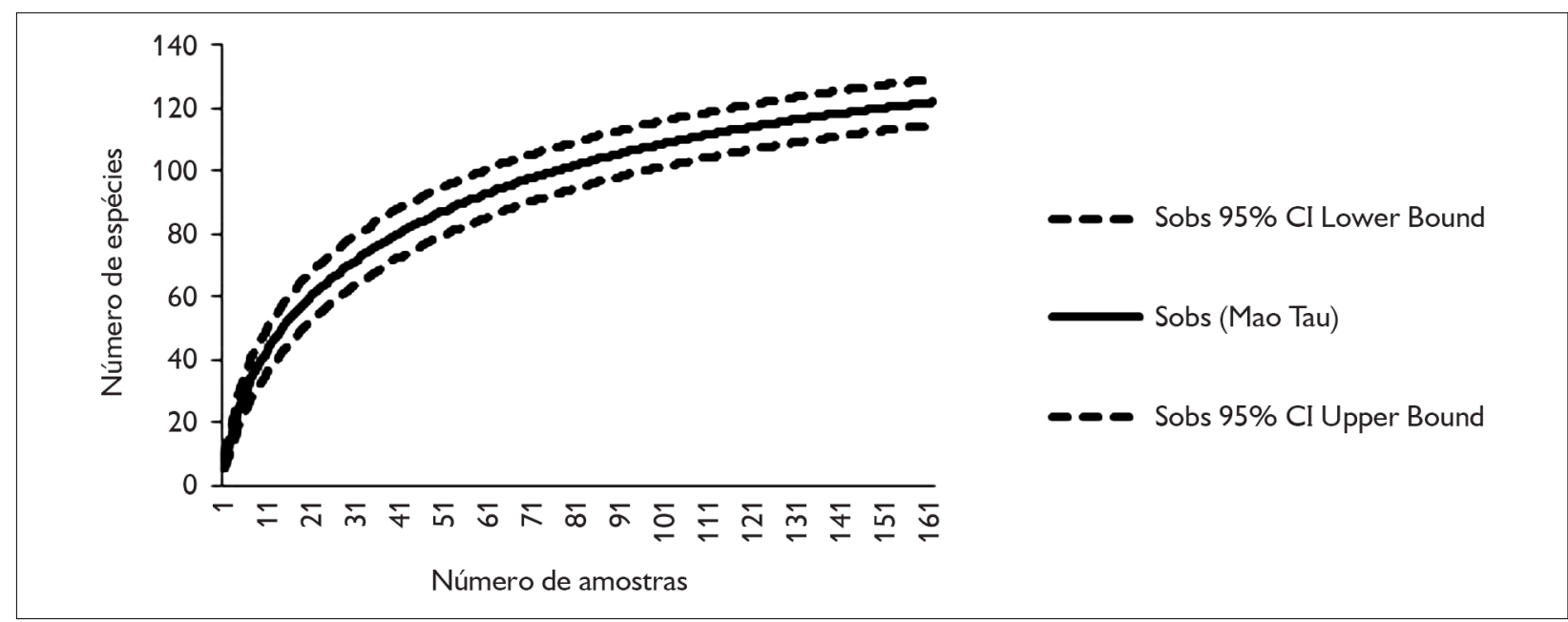

Figura 3. Curva de rarefação de espécies de fungos Agaricomycetes poroides em áreas florestais do município de Serra do Navio, estado do Amapá, Brasil.
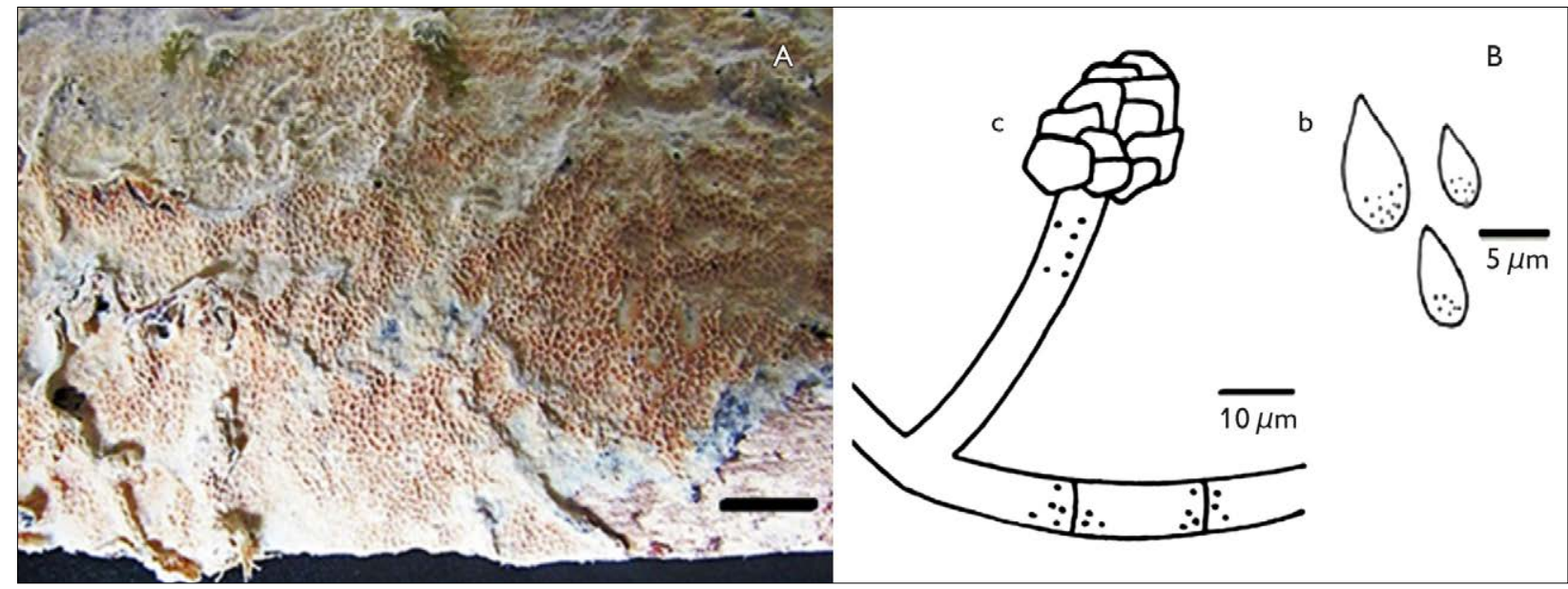

Figura 4. Basidioma e microestruturas de Oxyporus lacera coletado no município da Serra do Navio, estado do Amapá, Brasil: A) basidioma (barra de escala $=1 \mathrm{~cm}$ ); B) cistídio com cristais na região apical (c) e basidiósporos (b). Foto: William K. S. Xavier (2017) (A). Desenho: Adriene M. Soares (2018) (B).

\section{AGRADECIMENTOS}

Aos gestores e aos funcionários do Instituto Chico Mendes de Conservação da Biodiversidade (ICMBio), do Parque Montanhas do Tumucumaque, pelo apoio durante as viagens de campo; ao Programa de Pós-Graduação Rede de Biodiversidade e Biotecnologia da Amazônia Legal (Universidade Federal do Pará-Museu Paraense Emílio Goeldi - UFPA-MPEG); ao MPEG, à Universidade do Estado do Amapá (UEAP) e à Universidade Federal de Pernambuco (UFPE), pelo apoio logístico, oferecendo seus laboratórios e herbários.

\section{REFERÊNCIAS}

CENTRO DE REFERÊNCIAE INFORMAÇÃO AMBIENTAL (CRIA), [s. d.]. Specieslink. Disponível em: < http://www.splink.org.br/ index $>$. Acesso em: 20 fevereiro 2018.

COLWELL, R. K., 2009. EstimateS: statitical estimation of species richness and shared species from samples. Version 8.2. Disponível em: < http:// viceroy.eeb.uconn.edu/estimates >. Acesso em: 20 março 2018.

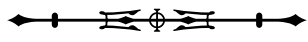


CROUS, P. W., M. J. WINGFIELD, T. I. BURGESS, G. E. S. J. HARDY, P. A. BARBER, P. ALVARADO, C. W. BARNES, P. K. BUCHANAN, M. HEYKOOP, G. MORENO, R. THANGAVEL, S. VAN DER SPUY, A. BARILI, S. BARRETT, S. O. CACCIOLA, J. F. CANO-LIRA, C. CRANE, C. DECOCK, T. B. GIBERTONI, J. GUARRO, M. GUEVARA-SUAREZ, V. HUBKA, M. KOLAŘÍK, C. R. S. LIRA, M. E. ORDOÑEZ, M. PADAMSEE, L. RYVARDEN, M. A. SOARES, A. M. STCHIGEL, D. A. SUTTON, A. VIZZINI, B. S. WEIR, K. ACHARYA, F. ALOI, I. G. BASEIA, R. A. BLANCHETTE, J. J. BORDALLO, Z. BRATEK, T. BUTLER, J. CANO-CANALS, J. R. CARLAVILLA, J. CHANDER, R. CHEEWANGKOON, R. H. S. F. CRUZ, M. DA SILVA, A. K. DUTTA, E. ERCOLE, V. ESCOBIO, F. ESTEVE-RAVENTÓS, J. A. FLORES, J. GENÉ, J. S. GÓIS, L. HAINES, B. W. HELD, M. HORTA JUNG, K. HOSAKA, T. JUNG, Ž. JURJEVIĆ, V. KAUTMAN, I. KAUTMANOVA, A. A. KIYASHKO, M. KOZANEK, A. KUBÁTOVÁ, M. LAFOURCADE, F. LA SPADA, K. P. D. LATHA, H. MADRID, E. F. MALYSHEVA, P. MANIMOHAN, J. L. MANJÓN, M. P. MARTÍN, M. MATA, Z. MERÉNYI, A. MORTE, I. NAGY, A. C. NORMAND, S. PALOI, N. PATTISON, J. PAWŁOWSKA, O. L. PEREIRA, M. E. PETTERSON, B. PICILLO, K. N. A. RAJ, A. ROBERTS, A. RODRÍGUEZ, F. J. RODRÍGUEZ-CAMPO, M. ROMAŃSKI, M. RUSZKIEWICZMICHALSKA, B. SCANU, L. SCHENA, M. SEMELBAUER, R. SHARMA, Y. S. SHOUCHE, V. SILVA, M. STANIASZEK-KIK, J. B. STIELOW, C. TAPIA, P. W. J. TAYLOR, M. TOOME-HELLER, J. M. C. VABEIKHOKHEI, A. D. VAN DIEPENINGEN, N. VAN HOA, M. VAN TRI, N. P. WIEDERHOLD, M. WRZOSEK, J. ZOTHANZAMA \& J. Z. GROENEWALD, 2017. Fungal Planet description sheets: 558-624. Persoonia 38: 240-384. DOI: <http:// dx.doi.org/10.3767/003158517X698941>.

DRUMMOND, J. A. \& M. A. PEREIRA, 2007. O Amapá nos tempos do manganês: um estudo sobre o desenvolvimento de um estado amazônico, 1943-2000: 1-498. Garamond, Rio de Janeiro.

FERREIRA-LOPES, V., G. L. ROBLEDO, M. A. RECK, A. GÓESNETO \& E. R. DRECHSLER-SANTOS, 2016. Phylloporia spathulata sensu stricto and two new South American stipitate species of Phylloporia (Hymenochaetaceae). Phytotaxa 257(2): 133-148. DOI: <http://dx.doi.org/10.11646/phytotaxa.257.2.3>.

FIDALGO, O. \& V. L. R. BONONI, 1989. Técnicas de coleta, preservação e herborização de material botânico: 1-62. Instituto de Botânica, São Paulo.

GIBERTONI, T. B., P. J. SANTOS \& M. A. CAVALCANTI, 2007. Ecological aspects of Aphyllophorales in the Atlantic rain forest in northeast Brazil. Fungal Diversity 25: 49-67.

GIBERTONI, T. B., 2008. Polyporoid fungi (Agaricomycetes, Basidiomycota) in the Estação Científica Ferreira Penna (State of Pará, Brazilian Amazonia): diversity and ecological aspects. Scientifica Acta 2(2): 70-74.

GIBERTONI, T. B., L. RYVARDEN, A. BERNICCHIA \& E. SAVINO, 2013. Poroid fungi (Agaricomycetes, Basidiomycota) in the National Caxiuanã Forest. In: P. L. B. LISBOA (Org.): Caxiuanã: paraíso ainda preservado: 397-409. Museu Paraense Emílio Goeldi, Belém.
GIBERTONI, T. B., P. S. MEDEIROS, A. M. S. SOARES, A. C. GOMES-SILVA, P. J. P. SANTOS, H. M. P. SOTÃO, L. V. FERREIRA \& E. SAVINO, 2016. The distribution of polypore fungi in endemism centres in Brazilian Amazonia. Fungal Ecology 20: 1-6. DOI: $<$ https://doi.org/10.1016/j.funeco.2015.09.012>

GOMES-SILVA, A. C. \& T. B. GIBERTONI, 2009. Checklist of the aphyllophoraceous fungi (Agaricomycetes) of the Brazilian Amazonia. Mycotaxon 108: 319-322.

HATTORI, T., 2005. Diversity of wood-inhabiting polypores in temperate forests with different vegetation types in Japan. Fungal Diversity 18: 73-88.

HIBBETT, D. S., R. BAUER, M. BINDER, A. J. GIACHINI, K. HOSAKA, A. JUSTO, E. LARSSON, K. H. LARSSON, J. D. LAWREY, O. MIETTINEN, L. G. NAGY, R. H. NILSSON, M. WEISS \& R. G. THORN, 2014. Agaricomycetes. In: D. J. MCLAUGHLIN \& J. W. SPATAFOR (Ed.): The mycota: systematics and evolution: 2. ed., Part A, v. 7: 373-429. Springer, Berlin, Heidelberg.

HYDE, K. D., C. NORPHANPHOUN, V. P. ABREU, A. BAZZICALUPO, K. W. THILINI CHETHANA, M. CLERICUZIO, M. C. DAYARATHNE, A. J. DISSANAYAKE, A. H. EKANAYAKA, M.-Q. HE, S. HONGSANAN, S.-K. HUANG, S. C. IAYASIRI, R. S. IAYAWARDENA, A. KARUNARATHNA, S. KONTA, I. KUSAN, H. LEE, J. LI, C.-G. LIN, N.-G. LIU, Y.-Z. LU, Z.-L. LUO, I. S. MANAWASINGHE, A. MAPOOK, R. H. PERERA, R. PHOOKAMSAK, C. PHUKHAMSAKDA, I. SIEDLECKI, A. M. SOARES, D. S. TENNAKOON, Q. TIAN, S. TIBPROMMA, D. N. WANASINGHE, Y.-P. XIAO, J. YANG, X.-Y. ZENG, F. A. ABDEL-AZIZ, W.-J. LI, I. C. SENANAYAKE, Q.-J. SHANG, D. A. DARANAGAMA, N. I. SILVA, K. M. THAMBUGALA, M. A. ABDEL-WAHAB, A. H. BAHKALI, M. L. BERBEE, S. BOONMEE, D. I. BHAT, T. S. BULGAKOV, B. BUYCK, E. CAMPORESI, R. F. CASTAÑEDA-RUIZ, P. CHOMNUNTI, M. DOILOM, F. DOVANA, T. B. GIBERTON, M. JADAN, R. JEEWON, E. B. G. JONES, J.-C. KANG, S. C. KARUNARATHNA, Y. W. LIM, J.-K. LIU, Z.-Y. LIU, H. L. PLAUTZJR., S. LUMYONG, S. S. N. MAHARACHCHIKUMBURA, N. MATOCEC, E. H. C. MCKENZIE, A. MESIC, D. MILLER, J. PAWŁOWSKA, O. L. PEREIRA, I. PROMPUTTHA, A. I. ROMERO, L. RYVARDEN, H.-Y. SU, S. SUETRONG, Z. TKALCEC, A. VIZZINI, T.-C. WEN, K. WISITRASSAMEEWON, M. WRZOSEK, J.-C. XU, Q. ZHAO, R.-L. ZHAO \& P. E. MORTIMER, 2017. Fungal diversity notes 603-708: taxonomic and phylogenetic notes on genera and species. Fungal Diversity 87(1): 1-235. DOI: < https://doi.org/10.1007/ s13225-017-0391-3>

INDEX FUNGORUM, 2018. Disponível em: <http://www. indexfungorum.org/>. Acesso em: 10 fevereiro 2018.

INSUMRAN, Y., U. KLINHOM \& P. PRAMUAL, 2012. Variability of internal transcribed spacer ribosomal DNA sequences of Fuscoporia gilva and Fuscoporia sp. in Thailand. Czech Mycology 64(1): 55-64.

JARDIM BOTÂNICO DO RIO DE JANEIRO (JBRJ), [s. d.]. Flora do Brasil 2020 em construção. Jardim Botânico do Rio de Janeiro, Rio de Janeiro. Disponível em: <http://floradobrasil.jbrj.gov.br>. Acesso em: 20 março 2018.

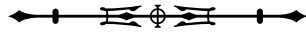


JUSTO, A., O. MIETTINEN, D. FLOUDAS, B. ORTIZ-SANTANA, E. SJOKVIST, D. LINDNER, K. NAKASONE, T. NIEMELA, K. LARSSON, L. RYVARDEN \& D. S. HIBBETT, 2017. A revised family-level classification of the Polyporales (Basidiomycota). Fungal Biology 121(9): 798-824. DOI: <https://doi.org/10.1016/.funbio.2017.05.010>

LINDBLAD, I., 2000. Host specificity of some wood-inhabiting fungi in a tropical forest. Mycologia 92(3): 399-405. DOI: <https://doi. org/10.2307/3761497>.

MEDEIROS, P. S., H. M. P. SOTÃO, T. B. GIBERTONI \& J. H. CATTANIO, 2013. Fungos poroides (Agaricomycetes) no sítio do Programa de Biodiversidade da Amazônia (PPBio) em Caxiuanã. In: P. L. B. LISBOA (Org.): Caxiuanã: paraíso ainda preservado: 375-385. Museu Paraense Emílio Goeldi, Belém.

PALACIO, M., G. L. ROBLEDO, M. A. RECK, E. GRASSI, A. GÓES-NETO \& E. R. DRECHSLER-SANTOS, 2017. Decrypting the Polyporus dictyopus complex: recovery of Atroporus Ryvarden and segregation of Neodictyopus gen. nov. (Polyporales, Basidiomyocta). PLOS ONE 12(10): e0186183. DOI: < https://doi.org/10.1371/journal. pone.0186183>.

PAUluS, B., N. HALLENBERG, P. K. BUCHANAN \& G. K. CHAMBERS, 2000. A phylogenetic study of the genus Schizopora (Basidiomycota) based on ITS DNA sequences. Mycological Research 104(10): 1155-1163. DOI: <https://doi.org/10.1017/ S0953756200002720>.

PIRES, R. M., V. MOTATO-VÁSQUEZ, M. C. WESTPHALEN \& A. M. GUGLIOTTA, 2017. Polyporales and similar poroid genera (Basidiomycota) from Parque Estadual da Serra do Mar, São Paulo State, Brazil. Hoehnea 44(1): 145-157. DOI: <http://dx.doi. org/10.1590/2236-8906-93/2016>.

ROBLEDO, G. L. \& M. RAJCHENBERG, 2007. South American polypores: first annotated checklist from Argentinean Yungas. Mycotaxon 100: 5-9.

RYARDEN, L. \& I. JOHANSEN, 1980. A preliminary polypore flora of East Africa: 1-636. Fungiflora, Oslo.

RYVARDEN, L., 1991. Genera of Polypores: nomenclature and taxonomy. Synopsis Fungorum 5(1): 1-373.

RYVARDEN, L. \& R. L. GILBERTSON, 1993. European Polypores. Synopsis Fungorum 6: 1-355.

RYARDEN, L., 2004. Neotropical polypores: Part 1: introduction, Ganodermataceae \& Hymenochaetaceae. Synopsis Fungorum 19: 1-227.

RYARDEN, L., 2007. Studies in Neotropical polypores. New and interesting wood-inhabiting fungi from Belize. Synopsis Fungorum 23: $32-50$.

RYVARDEN, L., 2014. Studies in Neotropical polypores 37. Some new and interesting species from tropical America. Synopsis Fungorum 32: 58-67.

RYVARDEN, L. \& I. MELO, 2014. Poroid fungi of Europe. Synopsis Fungorum 31: 1-455.
RYVARDEN, L., 2016. Neotropical polypores Part 3, Polyporaceae, Obba-Wrightoporia. Synopsis Fungorum 46: 445-613.

SOARES, A., H. SOTÃO, P. MEDEIROS \& T. GIBERTONI, 2014. Riqueza de fungos poliporoides (Agaricomycetes, Basidiomycota) em uma floresta ombrófila densa no Amapá, Amazônia brasileira. Boletim do Museu de Biologia Mello Leitão (Nova Série) 35: 5-18.

SOTÃO, H. M. P., V. L. R. BONONI \& T. S. FIGUEIREDO, 1991. Basidiomycetes de manguezais da ilha de Maracá, Amapá, Brasil. Boletim do Museu Paraense Emílio Goeldi, Série Botânica 7(1): 109-114.

SOTÃO, H. M. P., E. L. CAMPOS, A. M. GUGLIOTTA \& S. P. S. E. C. COSTA, 2003. Fungos macroscópicos: Basidiomycetes. In: M. E. B. FERNANDES (Ed.): Os manguezais da costa norte brasileira: $375-$ 385. Fundação Rio Bacanga, São Luís.

SOTÃO, H. M. P., T. B. GIBERTONI, R. MAZIERO, I. BASEIA, P. S. MEDEIROS, A. MARTINS-JÚNIOR \& M. CAPELARI, 2009. Fungos macroscópicos da Floresta Nacional de Caxiuanã, Pará, Brasil: Basidiomycota (Agaricomycetes). In: P. L. B. LISBOA (Ed.): Caxiuanã: paraíso ainda preservado: 383-396. Museu Paraense Emílio Goeldi, Belém.

TEIXEIRA, A. R., 1995. Método para estudo das hifas do basidiocarpo de fungos poliporáceos. Instituto de Botânica (Manual n. 6), São Paulo.

TIBPROMMA, S., K. D. HYDE, R. JEEWON, S. S. N MAHARACHCHIKUMBURA, J.-K. LIU, D. J. BHAT, E. B. G. JONES, E. H. C. MCKENZIE, E. CAMPORESI, T. S. BULGAKOV, M. DOILOM, A. L. C. AZEVEDO-SANTIAGO, K. MONTEIRO, P. MANIMOHAN, T. B. GIBERTONI, Y. W. LIM, A. H. EKANAYAKA, B. THONGBAI, H. B. LEE, J.-B. YANG, P. M. KIRK, P. SYSOUPHANTHONG, S. K. SINGH, S. BOONMEE, W. DONG, K. N. ANIL RAJ, K. P. L. DEEPNA, R. PHOOKAMSAK, C. PHUKHAMSAKDA, S. KONTA, S. C. JAYASIRI, C. NORPHANPHOUN, D. S. TENNAKOON, J. LI, M. C. DAYARATHNE, R. H. PERERA, Y. XIAO, D. N. WANASINGHE, I. C. SENANAYAKE, I. D. GOONASEKARA, N. I. SILVA, A. MAPOOK, R. S. JAYAWARDENA, A. J. DISSANAYAKE, I. S. MANAWASINGHE, T. K. W. CHETHANA, Z.-L. LUO, K. K. HAPUARACHCHI, A. BAGHELA, A. M. SOARES, A. VIZZINI, A. MEIRAS-OTTONI, A. MESIC, A. K. DUTTA, C. A. F. SOUZA, C. RICHTER, C.-G. LIN, D. CHAKRABARTY, D. A. DARANAGAMA, D. X. LIMA, D. CHAKRABORTY, E. ERCOLE, F.WU, G. SIMONINI, G. VASQUEZ, G. S. ALVES, H. L. PLAUTZ JR., H. A. ARIYAWANSA, H. LEE, I. KUSAN, J. SONG, J. SUN, J. KARMAKAR, K. HU, K. C. SEMWAL, K. M. THAMBUGALA, K. VOIGT, K. ACHARYA, K. C. RAJESHKUMAR, L. RYVARDEN, M. JADAN, M. I. HOSEN, M. MIKSIK, M. C. SAMARAKOON, N. N. WIJAYAWARDENE, N. K. KIM, N. MATOCEC, P. N. SINGH, Q. TIAN, R. P. BHATT, R. J. V. OLIVEIRA, R. E. TULLOSS, S. AAMIR, S. KAEWCHAI, S. D. MARATHE, S. KHAN, S. HONGSANAN, S. ADHIKARI, T. MEHMOOD, T. K. BANDYOPADHYAY, T. Y. SVETASHEVA, T. T. T. NGUYEN, V. ANTONIN, W.-J. LI, Y. WANG, Y. INDOLIYA, Z. TKALCEC, A. M. ELGORBAN, A. H. BAHKALI, A. M. C. TANG, H.Y. SU, H. ZHANG, I. PROMPUTTHA, J. LUANGSA-ARD, J. XU, J. YAN, K. JI-CHUAN, M. STADLER, P. E. MORTIMER, P. CHOMNUNTI, Q. ZHAO, A. J. L. PHILLIPS, S. NONTACHAIYAPOOM, T.-C. WEN \& S. C. KARUNARATHNA, 2017. Fungal diversity notes 491-602: taxonomic and phylogenetic contributions to fungal taxa. Fungal Diversity 83(1): 1-261. DOI: <https://doi.org/10.1007/s13225-017-0378-0>.

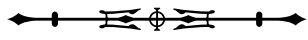


Apêndice. Lista de ordens, famílias e espécies de Agaricomycetes identificadas no município de Serra do Navio, estado do Amapá, Brasil, com informações sobre a classe de frequência e a indicação de novos registros $\left(*=\right.$ Amapá, ${ }^{*}=$ Amazônia, ${ }^{\bullet}=$ Brasil). Legendas: $A C=$

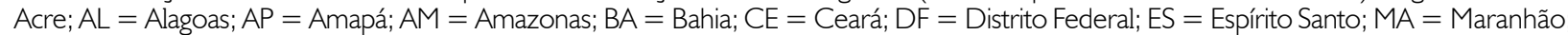
MT = Mato Grosso; MS = Mato Grosso do Sul; MG = Minas Gerais; PA = Pará; PB = Paraíba; PR = Paraná; PE = Pernambuco; PI = Piauí; RJ = Rio de Janeiro; RN = Rio Grande do Norte; RS = Rio Grande do Sul; RO = Rondônia; RR = Roraima; $S C=$ Santa Catarina; SE = Sergipe, SP = São Paulo; TO = Tocantins; literatura: ${ }^{1}=$ CRIA (s. d.), ${ }^{2}=$ Crous et al. (2017), ${ }^{3}=$ Hyde et al. $(2017),{ }^{4}=$ JBRJ (s. d.), ${ }^{5}=$ Pires et al. (2017), ${ }^{6}=$ Soares et al. (2014).

\begin{tabular}{|c|c|c|c|c|}
\hline Ordem/Família/Espécies & $\begin{array}{l}\text { Classes de } \\
\text { frequência }\end{array}$ & $\begin{array}{l}\text { Número de } \\
\text { espécimes } \\
\text { identificados }\end{array}$ & Voucher & Distribuição no Brasil \\
\hline \multicolumn{5}{|l|}{ Hymenochaetales } \\
\hline \multicolumn{5}{|l|}{ Hymenochaetaceae Donk } \\
\hline Coltricia barbata Ryvarden \& de Meijer & Rara & 1 & MG226141 & AP, PA, PR, RO, SC $1,4,6$ \\
\hline $\begin{array}{l}\text { Fomitiporella cavicola (Kotl. \& Pouzar) } \\
\text { T. Wagner \& M. Fisch }\end{array}$ & Rara & 8 & MG226116/HAMAB019112 & $\begin{array}{c}\mathrm{AL}, \mathrm{AP}, \mathrm{BA}, \mathrm{PA}, \mathrm{PE}, \mathrm{PR}, \mathrm{RN} \\
\mathrm{RS}, \mathrm{SE}, \mathrm{SC}, \mathrm{SP}^{1,4}\end{array}$ \\
\hline $\begin{array}{l}\text { Fomitiporia baccharidis (Pat.) Decock, } \\
\text { Robledo \& Amalfi }\end{array}$ & Rara & 2 & MG226113 & $\begin{array}{l}\text { AC, } A P, A L, B A, P A, P B \\
\text { PE, PI, RN } \\
\text { R } 4,6\end{array}$ \\
\hline Fomitiporia punctata (P. Karst.) Murrill & Rara & 1 & MG226109 & AP, PR, RS, SC, SP', 4 \\
\hline $\begin{array}{l}\text { Fulvifomes membranaceus (J.E. Wright } \\
\text { \& Blumenf.) Baltazar \& Gibertoni* }\end{array}$ & Rara & 1 & MG226154 & $\mathrm{AM}, \mathrm{BA}, \mathrm{PA}, \mathrm{PE}, \mathrm{RO}, \mathrm{RS}^{1,4}$ \\
\hline $\begin{array}{l}\text { Fuscoporia callimorpha (Lév.) Groposo, } \\
\text { Log.-Leite \& Góes-Neto sensu lato }\end{array}$ & Ocasional & 40 & MG226096/HAMAB019078 & $\begin{array}{l}\text { AP, MT, PA, PB, PE, } \\
\text { RO, SC, SP, } 4\end{array}$ \\
\hline Fuscoporia contigua (Pers.) G. Cunn.* & Rara & 3 & MG227460 & $\mathrm{AL}, \mathrm{BA}, \mathrm{PA}, \mathrm{PE}, \mathrm{SC}, \mathrm{RO}, \mathrm{RS}^{1,4}$ \\
\hline $\begin{array}{l}\text { Fuscoporia undulata (Murrill) Bondartseva } \\
\& \text { S. Herrera }\end{array}$ & Rara & 12 & MG226127/HAMAB019113 & $\mathrm{AL}, \mathrm{AP}, \mathrm{PA}, \mathrm{PB}, \mathrm{SC} \mathrm{C}^{1,4}$ \\
\hline $\begin{array}{l}\text { Hymenochaete iodina (Mont.) Baltazar \& } \\
\text { Gibertoni }\end{array}$ & Rara & 15 & MG226117/HAMAB019110 & $\begin{array}{c}\text { AC, AM, AP, BA, ES, MT, } \\
\text { PA, PE, PR, RO, RR, SC, SP', } 4,6\end{array}$ \\
\hline $\begin{array}{l}\text { Inonotus calcitratus (Berk. \& M.A. Curtis) } \\
\text { Gomes-Silva \& Gibertoni }\end{array}$ & Rara & 4 & MG226092/HAMAB019105 & AM, AP, BA, PA, PE, PI, RO, RS $1,4,6$ \\
\hline Phellinidium lamaoense (Murrill) Y.C. Dai* & Rara & 1 & MG227459 & $\mathrm{PA}^{4}$ \\
\hline Phellinus anchietanus Decock \& Ryvarden & Rara & 1 & MG226146 & $\mathrm{SC}^{4}$ \\
\hline Phellinus fastuosus (Lév.) S. Ahmad & Rara & 9 & MG226153/HAMAB019104 & $\begin{array}{l}\text { AL, AM, AP, PA, PB, PR, } \\
\text { RN, RR, RS, SC, SP', 4,6 }\end{array}$ \\
\hline Phellinus shaferi (Murrill) Ryvarden & Rara & 3 & MG226103 & $\mathrm{AL}, \mathrm{AP}, \mathrm{BA}, \mathrm{PA}, \mathrm{PE}, \mathrm{PI}^{1,4}$ \\
\hline $\begin{array}{l}\text { Phylloporia spathulata (Hook.) Ryvarden } \\
\text { sensu lato }\end{array}$ & Ocasional & 40 & MG226140/HAMAB019072 & $\begin{array}{l}\text { AM, AP, BA, MG, PA, PB, PE, } \\
P R, R O, R R, R S, S C, S^{1,4,6}\end{array}$ \\
\hline $\begin{array}{l}\text { Tropicoporus dependens (Murrill) L.W. } \\
\text { Zhou, Y.C. Dai \& Vlasák }\end{array}$ & Rara & 4 & MG226098 & $\mathrm{AM}, \mathrm{AP}, \mathrm{PA}, \mathrm{PE}, \mathrm{RS}^{1,4}$ \\
\hline $\begin{array}{l}\text { Tropicoporus linteus (Berk. \& M.A. Curtis) } \\
\text { L.W. Zhou \& Y.C. Dai* }\end{array}$ & Rara & 2 & MG226138 & $\begin{array}{c}\mathrm{AL}, \mathrm{BA}, \mathrm{CE}, \mathrm{PA}, \mathrm{PB}, \mathrm{PE}, \mathrm{PI} \\
\mathrm{PR}, \mathrm{RR}, \mathrm{SC}^{1,4}\end{array}$ \\
\hline \multicolumn{5}{|l|}{ Schizoporaceae Jülich } \\
\hline $\begin{array}{l}\text { Xylodon flaviporus (Berk. \& M.A. Curtis } \\
\text { ex Cooke) Riebesehl \& Langer sensu lato }\end{array}$ & Frequente & 130 & MG226089/HAMAB019086 & $\begin{array}{l}\text { AC, AM, AP, CE, PA, PB, } \\
\text { PE, RJ, RN, RS, SC } 1,4,6\end{array}$ \\
\hline \multicolumn{5}{|l|}{ Incertae sedis } \\
\hline Trichaptum biforme (Fr.) Ryvarden* & Rara & 1 & HAMAB01912 & $\begin{array}{l}\mathrm{AL}, \mathrm{AM}, \mathrm{BA}, \mathrm{MA}, \mathrm{MT}, \mathrm{PA}, \mathrm{PB}, \mathrm{PE} \\
\mathrm{PI}, \mathrm{PR}, \mathrm{RJ}, \mathrm{RO}, \mathrm{RS}, \mathrm{SC}, \mathrm{SP}, 4\end{array}$ \\
\hline
\end{tabular}


Apêndice.

(Continua)

\begin{tabular}{|c|c|c|c|c|}
\hline \\
\hline Ordem/Família/Espécies & $\begin{array}{l}\text { Classes de } \\
\text { frequência }\end{array}$ & $\begin{array}{l}\text { Número de } \\
\text { espécimes } \\
\text { identificados }\end{array}$ & Voucher & Distribuição no Brasil \\
\hline $\begin{array}{l}\text { Trichaptum griseofuscum (Mont.) } \\
\text { Ryvarden \& Iturr. }\end{array}$ & Rara & 3 & MG226156 & $\begin{array}{c}A C, A M, A P, B A, M T, P A \\
P B, R O, R^{1,6}\end{array}$ \\
\hline Trichaptum perrottetii (Lév.) Ryvarden & Rara & 5 & MG226090/HAMAB019114 & AC, AM, AP, PA, RO, RR', 4,6 \\
\hline Trichaptum sector (Ehrenb.) Kreisel & Rara & 3 & MG226080/HAMAB019094 & $\begin{array}{c}\text { AM, AP, BA, PA, PB, PE, PR, RJ, } \\
\text { RO, RR, RS, SC, SP1, 4,6 }\end{array}$ \\
\hline \multicolumn{5}{|l|}{ Polyporales } \\
\hline \multicolumn{5}{|l|}{ Fomitopsidaceae Jülich } \\
\hline $\begin{array}{l}\text { Daedalea dochmia (Berk. \& Broome) } \\
\text { T. Hatt.* }\end{array}$ & Rara & 2 & MG226110 & $\mathrm{AM}, \mathrm{DF}, \mathrm{RO}{ }^{1}$ \\
\hline $\begin{array}{l}\text { Fomitopsis roseoalba A.M.S. Soares, } \\
\text { Ryvarden \& Gibertoni }\end{array}$ & Ocasional & 47 & MG227454/HAMAB019115 & $A P, P A^{1}$ \\
\hline Fomitopsis scalaris (Cooke) Ryvarden & Rara & 6 & MG226074/HAMAB019119 & AP, AM, PA, RO, RR 1,4 \\
\hline Fomitella supina (Sw.) Murrill & Rara & 2 & MG226129 & $\begin{array}{c}\mathrm{AL}, \mathrm{AP}, \mathrm{BA}, \mathrm{PA}, \mathrm{PB}, \mathrm{PE}, \mathrm{PR} \\
\mathrm{RS}, \mathrm{SE}, \mathrm{SC}, \mathrm{SP}^{1,4}\end{array}$ \\
\hline \multicolumn{5}{|l|}{ Ganodermataceae Donk } \\
\hline $\begin{array}{l}\text { Amauroderma camerarium (Berk.) } \\
\text { J. S. Furtado }\end{array}$ & Rara & 4 & MG226142 & $\begin{array}{l}\text { AP, AM, BA, MS, MT, PA, PE, } \\
\text { PR, RJ, RS, SE, SC } 1,4,6\end{array}$ \\
\hline $\begin{array}{l}\text { Amauroderma intermedium (Bres. \& Pat.) } \\
\text { Torrend }\end{array}$ & Rara & 4 & MG226115 & $\begin{array}{l}\text { AM, AP, BA, PA, PE, PR, } \\
\text { RJ, SC, SP'1, } 4\end{array}$ \\
\hline $\begin{array}{l}\text { Amauroderma schomburgkii (Mont. \& } \\
\text { Berk.) Torrend }\end{array}$ & Ocasional & 42 & MG226105/HAMAB019080 & $\begin{array}{l}\text { AM, AP, BA, MG, PA, PE, PR, } \\
\text { RJ, RO, RS, SC, SE, SP' } 4,6\end{array}$ \\
\hline Ganoderma australe (Fr.) Pat. & Ocasional & 63 & MG226122/HAMAB019073 & $\begin{array}{c}\text { AC, AM, AP, BA, MG, MS, PA, PE, } \\
\text { PR, RJ, RO, RR, RS, SC, SP } 1,4,6\end{array}$ \\
\hline Ganoderma multiplicatum (Mont.) Pat. & Rara & 2 & MG226073 & $\begin{array}{c}A L, A M, A P, M S, P A, R J, R O \\
\text { RR, SC, SE, SP }\end{array}$ \\
\hline Ganoderma orbiforme (Fr.) Ryvarden* & Rara & 1 & MG226095 & $\begin{array}{c}\text { AM, BA, CE, MS, PE, PR, } \\
\text { RJ, RO, SP1, } 4\end{array}$ \\
\hline Ganoderma stipitatum (Murrill) Murrill & Rara & 5 & MG226087/HAMAB019091 & $A C, A M, A P, C E, P A, P B, P E, R O^{1,4}$ \\
\hline \multicolumn{5}{|l|}{ Meripilaceae Jülich } \\
\hline Rigidoporus amazonicus Ryvarden & Rara & 1 & MG227456 & $\mathrm{AM}, \mathrm{AP}, \mathrm{PA}, \mathrm{RO}, \mathrm{SC}^{1,4,6}$ \\
\hline $\begin{array}{l}\text { Rigidoporus biokoensis (Bres. ex Lloyd) } \\
\text { Ryvarden }\end{array}$ & Rara & 17 & MG227462/HAMAB019116 & AC, AP, BA, MA, PA, PE, RO',4,6 \\
\hline Rigidoporus microporus (Sw.) Overeem & Rara & 11 & MG226099/HAMAB019079 & $\begin{array}{c}\text { AC, AM, AP, PA, PE, RO, RR, } \\
\text { RS, SC, SP } \mathrm{SP}^{1,4} 6\end{array}$ \\
\hline Rigidoporus undatus (Pers.) Donk & Rara & 11 & MG226085/HAMAB019092 & AP, PA, SP, RS, SC, SP1, 4 \\
\hline Rigidoporus vinctus (Berk.) Ryvarden & Ocasional & 43 & MG226120/HAMAB019103 & $\begin{array}{l}\text { AC, AM, AP, PA, RO, } \\
\text { RR, SC, SP1, } 4,6\end{array}$ \\
\hline \multicolumn{5}{|l|}{ Meruliaceae Rea } \\
\hline $\begin{array}{l}\text { Flaviporus hydrophilus (Berk. \& M.A. } \\
\text { Curtis) Ginns }\end{array}$ & Rara & 2 & MG226071/HAMAB019096 & $\mathrm{AL}, \mathrm{AP}, \mathrm{BA}, \mathrm{PB}, \mathrm{PE}, \mathrm{PR}, \mathrm{SC}, \mathrm{SP}^{1,4,6}$ \\
\hline
\end{tabular}


Apêndice.

(Continua)

\begin{tabular}{|c|c|c|c|c|}
\hline Ordem/Família/Espécies & $\begin{array}{l}\text { Classes de } \\
\text { frequência }\end{array}$ & $\begin{array}{l}\text { Número de } \\
\text { espécimes } \\
\text { identificados }\end{array}$ & Voucher & Distribuição no Brasil \\
\hline Flaviporus liebmannii (Fr.) Ginns & Rara & 6 & MG227457/HAMAB019118 & $\begin{array}{c}\text { AM, AP, BA, PA, PR, RO, } \\
\text { RS, SC, SP1, 4, }\end{array}$ \\
\hline Junghuhnia carneola (Bres.) Rajchenb. & Rara & 6 & MG226107/HAMAB019085 & $\mathrm{AM}, \mathrm{AP}, \mathrm{PA}, \mathrm{PR}, \mathrm{RS}, \mathrm{SC}, \mathrm{SP}^{1,4,6}$ \\
\hline Junghuhnia subundata (Murrill) Ryvarden & Rara & 13 & MG226133/HAMAB019117 & $A M, A P, R O^{1,6}$ \\
\hline \multicolumn{5}{|l|}{ Phanerochaetaceae Jülich } \\
\hline Antrodiella murrillii (Lloyd) Ryvarden* & Rara & 1 & MG226135 & $A C, A M, M T, P A^{1}$ \\
\hline $\begin{array}{l}\text { Ceriporia amazonica A.M.S. Soares, H.M.P. } \\
\text { Sotão \& Ryvarden }\end{array}$ & Rara & 3 & MG226151/HAMAB019106 & AP, $\mathrm{PA}^{1,4}$ \\
\hline Ceriporia purpurea (Fr.) Donk $\bullet$ & Rara & 2 & MG227465 & $\mathrm{RS}^{4}$ \\
\hline $\begin{array}{l}\text { Ceriporia xylostromatoides (Berk.) } \\
\text { Ryvarden* }\end{array}$ & Rara & 1 & MG227464 & $\mathrm{AC}, \mathrm{AM}, \mathrm{PR}, \mathrm{RR}, \mathrm{RS}, \mathrm{SC}, \mathrm{SP}^{1,4}$ \\
\hline \multicolumn{5}{|l|}{ Polyporaceae Corda } \\
\hline Abundisporus roseoalbus (Jungh.) Ryvarden & Rara & 4 & MG226104 & $\begin{array}{c}\text { AC, AP, BA, PA, PE, PR, } \\
\text { RO, RS, SC } 1,4,6\end{array}$ \\
\hline Coriolopsis brunneoleuca (Berk.) Ryvarden & Rara & 2 & MG226139 & AP, AM, PA 1,6 \\
\hline $\begin{array}{l}\text { Dichomitus amazonicus Gomes-Silva, } \\
\text { Ryvarden \& Gibertoni }\end{array}$ & Ocasional & 37 & MG226093/HAMAB019095 & AM, AP, BA, CE, PA, PR 14,6 \\
\hline $\begin{array}{l}\text { Dichomitus anoectoporus (Berk. \& M.A. } \\
\text { Curtis) Ryvarden* }\end{array}$ & Rara & 4 & MG226157 & PA, PI, RS, SC 1,4 \\
\hline $\begin{array}{l}\text { Dichomitus campestris (Quél.) Domanski } \\
\& \text { Orlicz }\end{array}$ & Rara & 1 & MG227463 & $\mathrm{SP}^{5}$ \\
\hline $\begin{array}{l}\text { Diplomitoporus allantosporus Ryvarden \& } \\
\text { Iturr.* }\end{array}$ & Rara & 6 & MG226136/HAMAB019099 & $\mathrm{PA}, \mathrm{SP}^{1,4}$ \\
\hline $\begin{array}{l}\text { Earliella scabrosa (Pers.) Gilb. \& } \\
\text { Ryvarden }\end{array}$ & Ocasional & 51 & MG226088/HAMAB019066 & $\begin{array}{l}\mathrm{AC}, \mathrm{AL}, \mathrm{AM}, \mathrm{AP}, \mathrm{BA}, \mathrm{PA}, \mathrm{PB}, \mathrm{PE} \\
\mathrm{PR}, \mathrm{RO}, \mathrm{RR}, \mathrm{SC}, \mathrm{SP}, \mathrm{TO}^{1,4,6}\end{array}$ \\
\hline Favolus tenuiculus P. Beauv. & Rara & 12 & MG226084/HAMAB019089 & $\begin{array}{c}\text { AC, AM, AP, AL, BA, CE, MS, MT, } \\
\text { MG, PA, PB, PE, PR, RN, RJ, RS, } \\
\text { RO, RR, SE, SP, SC, } 1,6\end{array}$ \\
\hline Fomes extensus (Lév.) Cooke & Rara & 2 & MG226134 & AM, AP, BA, PE, RR, RS, SP, TO ${ }^{1,4}$ \\
\hline $\begin{array}{l}\text { Funalia aspera (Jungh.) Zmitr. \& } \\
\text { Malysheva* }\end{array}$ & Rara & 4 & MG226119 & AM, MG, SP, RS, SC 1,4 \\
\hline $\begin{array}{l}\text { Funalia caperata (Berk.) Zmitr. \& } \\
\text { Malysheva }\end{array}$ & Ocasional & 31 & MG226081/HAMAB019071 & $\begin{array}{l}\text { AC, AL, AM, AP, BA, MG, MT, } \\
\text { PA, PB, PE, PI, PR, RJ, RN, RO, } \\
\text { RR, RS, SE, SC, SP, TO } 1,4,6\end{array}$ \\
\hline $\begin{array}{l}\text { Grammothele fuligo (Berk. \& Broome) } \\
\text { Ryvarden }\end{array}$ & Rara & 10 & MG226069/HAMAB019108 & AP, AM, PA, PE, RO, RS, SC', 4,6 \\
\hline Grammothele lineata Berk. \& M.A. Curtis & Rara & 7 & MG226145/HAMAB019120 & $\mathrm{AL}, \mathrm{AM}, \mathrm{AP}, \mathrm{PE}, \mathrm{RR}, \mathrm{RS}, \mathrm{SC}, \mathrm{SP}^{1,4,6}$ \\
\hline $\begin{array}{l}\text { Grammothele subargentea (Speg.) } \\
\text { Rajchenb. }\end{array}$ & Rara & 17 & MG226130/HAMAB019121 & $\begin{array}{l}\mathrm{AL}, \mathrm{AP}, \mathrm{PB}, \mathrm{PE}, \mathrm{PR}, \mathrm{RR} \\
\mathrm{RS}, \mathrm{SC}, \mathrm{SP}^{1,4,6}\end{array}$ \\
\hline Lentinus swartzii Berk. & Rara & 11 & MG226144/HAMAB019069 & AC, AM, AP, PA, RO, RR, TO', 4 \\
\hline
\end{tabular}


Apêndice.

(Continua)

\begin{tabular}{|c|c|c|c|c|}
\hline Ordem/Família/Espécies & $\begin{array}{l}\text { Classes de } \\
\text { frequência }\end{array}$ & $\begin{array}{l}\text { Número de } \\
\text { espécimes } \\
\text { identificados }\end{array}$ & Voucher & Distribuição no Brasil \\
\hline $\begin{array}{l}\text { Leiotrametes lactinea (Berk.) Welti \& } \\
\text { Courtec. }\end{array}$ & Rara & 2 & HAMAB019126 & $\begin{array}{l}\text { AC, } A M, B A, M S, P A, P E \\
\text { RO, RR, RS, SE }\end{array}$ \\
\hline Loweporus tephroporus (Mont.) Ryvarden * & Rara & 4 & MG226158/HAMAB019102 & PA, PE, PI, RO, SC, SP1, 4 \\
\hline $\begin{array}{l}\text { Megasporoporia setulosa (Henn.) } \\
\text { Rajchenb.* }\end{array}$ & Ocasional & 29 & MG226121/HAMAB019093 & $\begin{array}{l}\text { AM, CE, PA, PI, PR, RO, } \\
\text { RR, RS, SC, SP1, } 4\end{array}$ \\
\hline $\begin{array}{l}\text { Megasporoporiella cavernulosa (Berk.) B.K. } \\
\text { Cui, Y.C. Dai \& Hai J. Li }\end{array}$ & Ocasional & 25 & MG226070/HAMAB019084 & $\begin{array}{c}\mathrm{AL}, \mathrm{AM}, \mathrm{AP}, \mathrm{BA}, \mathrm{PA}, \mathrm{PE}, \mathrm{PR}, \mathrm{RN} \\
\mathrm{RO}, \mathrm{RR}, \mathrm{RS}, \mathrm{SC}, \mathrm{SE}, \mathrm{SP}^{1,4,6}\end{array}$ \\
\hline Microporellus obovatus (Jungh.) Ryvarden & Ocasional & 29 & MG226111/HAMAB019065 & $\begin{array}{c}\mathrm{AL}, \mathrm{AM}, \mathrm{AP}, \mathrm{BA}, \mathrm{PA}, \mathrm{PB}, \mathrm{PE}, \mathrm{PR} \\
\mathrm{RN}, \mathrm{RO}, \mathrm{RS}, \mathrm{SC}, \mathrm{SE}, \mathrm{SP}^{1,4,6}\end{array}$ \\
\hline Navisporus sulcatus (Lloyd) Ryvarden & Rara & 2 & MG226118 & $A C, A M, P R, R R, R S^{1,4}$ \\
\hline Nigrofomes melanoporus (Mont.) Murrill & Rara & 2 & MG226094 & $\begin{array}{c}\mathrm{AL}, \mathrm{AP}, \mathrm{BA}, \mathrm{PA}, \mathrm{PB}, \mathrm{PE}, \mathrm{PR} \\
\mathrm{RO}, \mathrm{RR}, \mathrm{SP}^{1,4,6}\end{array}$ \\
\hline Nigroporus vinosus (Berk.) Murrill & Ocasional & 20 & MG226100/HAMAB019090 & $\begin{array}{l}\text { AC, } A P, M T, P B, P E, ~ R N \\
\text { RO, RS, SC, } S P^{1,4,6}\end{array}$ \\
\hline $\begin{array}{l}\text { Pachykytospora alabamae (Berk. et Cke.) } \\
\text { Ryvarden* }\end{array}$ & Rara & 4 & MG227458 & AM, PA, PE, PR, RR, RS, SC, SP1, 4 \\
\hline $\begin{array}{l}\text { Perenniporia brasiliensis Lira, A.M.S. } \\
\text { Soares, Ryvarden \& Gibertoni }\end{array}$ & Rara & 3 & HAMAB019123 & $A P^{2}$ \\
\hline $\begin{array}{l}\text { Perenniporia centrali-africana Decock \& } \\
\text { Mossebo }\end{array}$ & Rara & 2 & MG226147 & $P E^{1}$ \\
\hline $\begin{array}{l}\text { Perenniporia contraria (Berk. \& M.A. } \\
\text { Curtis) Ryvarden }\end{array}$ & Rara & 3 & MG226086/HAMAB019083 & AL, AP, PA, PE, PR, RO, SC $1,4,6$ \\
\hline $\begin{array}{l}\text { Perenniporia cremeopora Decock \& } \\
\text { Ryvarden }\end{array}$ & Rara & 2 & MG226091 & AP, PA, PE $1,4,6$ \\
\hline Perenniporia inflexibilis (Berk.) Ryvarden & Rara & 8 & MG226083/HAMAB019068 & AM, AP, BA, MT, PA, RO, RR $1,4,6$ \\
\hline Perenniporia martia (Berk.) Ryvarden & Rara & 8 & MG227455 & $\begin{array}{l}\text { AM, AP, BA, MS, MT, PA, PB, PE, } \\
\text { PR, RN, RO, RS, SC, SP1, 4,6 }\end{array}$ \\
\hline $\begin{array}{l}\text { Perenniporia parvispora Decock \& } \\
\text { Ryvarden* }\end{array}$ & Rara & 1 & HАMАВ019124 & $\mathrm{PA}, \mathrm{PR}, \mathrm{SP}^{1,4}$ \\
\hline $\begin{array}{l}\text { Perenniporia roseoisabellina (Pat. \& } \\
\text { Gaillard) Ryvarden }\end{array}$ & Rara & 1 & MG226079 & AP, $\mathrm{PA}^{1,4}$ \\
\hline Perenniporia stipitata Ryvarden & Ocasional & 30 & MG226125/HAMAB019077 & AP, PA, RO, SC $1,4,6$ \\
\hline Polyporus dictyopus Mont. sensu lato & Ocasional & 64 & MG226114/HAMAB019075 & $\begin{array}{c}\text { AC, } A M, A P, B A, C E, P A, P B, P E \\
\text { RO, RR, RS, SC, SP1, 4, }\end{array}$ \\
\hline Polyporus guianensis Mont. & Ocasional & 53 & MG226102/HAMAB019070 & $A C, A M, A P, P A^{1,4,6}$ \\
\hline Polyporus leprieurii Mont. & Ocasional & 73 & MG226097/HAMAB019081 & $\begin{array}{c}\text { AC, } A M, A P, C E, P A, P B, P E, P R \\
\text { RJ, RO, RS, SC, SP1, } 4,6\end{array}$ \\
\hline $\begin{array}{l}\text { Porogramme albocincta (Cooke \& } \\
\text { Massee) Gibertoni }\end{array}$ & Ocasional & 47 & MG226148/HAMAB019063 & AP, PA, PE, SC, SP1, 4,6 \\
\hline
\end{tabular}


Apêndice.

(Conclusão)

\begin{tabular}{|c|c|c|c|c|}
\hline Ordem/Família/Espécies & $\begin{array}{l}\text { Classes de } \\
\text { frequência }\end{array}$ & $\begin{array}{l}\text { Número de } \\
\text { espécimes } \\
\text { identificados }\end{array}$ & Voucher & Distribuição no Brasil \\
\hline Pycnoporus sanguineus (L.) Murrill & Rara & 2 & MG226076 & $\begin{array}{c}\text { AC, AM, AP, BA, CE, ES, MA, MT, } \\
\text { PA, PB, RO, RN, RS, SC, SP', }\end{array}$ \\
\hline Pyrofomes lateritius (Cooke) Ryvarden & Rara & 10 & MG226075/HAMAB019111 & $\begin{array}{c}\text { AM, AP, BA, PA, PR, RJ, } \\
\text { RO, RS, SC } \mathrm{SC}^{1,4,6}\end{array}$ \\
\hline $\begin{array}{l}\text { Tinctoporellus epimiltinus (Berk. \& } \\
\text { Broome) Ryvarden }\end{array}$ & Ocasional & 72 & MG227466/HAMAB019064 & $\begin{array}{l}\text { AP, PA, BA, CE, DF, MT, PE, SP, } \\
\text { RS, RO, RR, SC, SP1, 4,6 }\end{array}$ \\
\hline Trametes elegans (Spreng.) Fr. & Ocasional & 80 & MG226077/HAMAB019067 & $\begin{array}{l}\text { AC, AM, AP, BA, MT, PA, PE, } \\
\text { PR, RJ, RO, RS, SC, SP } 14,6\end{array}$ \\
\hline Trametes membranacea (Sw.) Kreisel & Rara & 3 & MG226078 & AM, AP, PA, PB, PE, RJ, RS, SC',4 \\
\hline $\begin{array}{l}\text { Trametes modesta (Kunzeex Fr.) } \\
\text { Ryvarden }\end{array}$ & Ocasional & 54 & MG226082/HAMAB019082 & $\begin{array}{c}A C, A M, A P, B A, M A, M T \\
P A, R O, R^{1,4,6}\end{array}$ \\
\hline Trametes nivosa (Berk.) Murrill & Rara & 1 & HAMAB019127 & $\begin{array}{l}\text { AM, AP, BA, DF, PA, PE, PI, } \\
\text { RJ, RO, SC, SP } 1,4,6\end{array}$ \\
\hline Trametes psila (Lloyd) Ryvarden & Rara & 1 & MG226143 & $\mathrm{AM}, \mathrm{AP}, \mathrm{BA}, \mathrm{RO}, \mathrm{RR}^{1,6}$ \\
\hline Trametes supermodesta Ryvarden \& Iturr. & Rara & 1 & MG226128 & $\begin{array}{l}\text { AM, AP, CE, MT, PA, PB, } \\
\text { PE, PI, RO, RR', }\end{array}$ \\
\hline \multicolumn{5}{|l|}{ Steccherinaceae Parmasto } \\
\hline $\begin{array}{l}\text { Trulla dentipora (Ryvarden \& Iturr.) } \\
\text { Miettinen \& Ryvarden }\end{array}$ & Rara & 4 & MG226108/HAMAB019098 & $A P^{1}$ \\
\hline Trulla meridae Miettinen \& Ryvarden & Rara & 2 & HAMAB019125 & $\mathrm{AP}^{3}$ \\
\hline \multicolumn{5}{|l|}{ Polyporales - Incertae sedis } \\
\hline Oxyporus lacera Ryvarden & Rara & 1 & MG226149 & \\
\hline $\begin{array}{l}\text { Oxyporus latemarginatus (Durieu \& } \\
\text { Mont.) Donk }\end{array}$ & Rara & 2 & MG226152 & $\mathrm{SC}, \mathrm{SP}^{1}$ \\
\hline Oxyporus mollis Gibertoni \& Ryvarden & Rara & 3 & MG226155 & $\mathrm{AP}, \mathrm{RJ}{ }^{1}$ \\
\hline $\begin{array}{l}\text { Rickiopora latemarginata (Rick) } \\
\text { Westphalen, Tomšovský \& Rajchenb.* }\end{array}$ & Rara & 4 & MG226131 & $\mathrm{PE}, \mathrm{RS}, \mathrm{SC}, \mathrm{SP}^{1}$ \\
\hline \multicolumn{5}{|l|}{ Russulales } \\
\hline \multicolumn{5}{|l|}{ Bondarzewiaceae Kotl. \& Pouzar } \\
\hline Wrightoporia avellanea (Bres.) Pouzar & Rara & 1 & MG226101 & AP, BA, CE, PA, SC, SP1 \\
\hline \multicolumn{5}{|l|}{ Incertae sedis } \\
\hline $\begin{array}{l}\text { Larssoniporia tropicalis (Cooke) Y.C. Dai, } \\
\text { Jia J. Chen \& B.K. Cui }\end{array}$ & Rara & 7 & MG226132/HAMAB019107 & $A P, B A^{1,6}$ \\
\hline \multicolumn{2}{|l|}{ TOTAL: 100} & 1.443 & & \\
\hline
\end{tabular}


